\title{
Determination of Candida species nestled in denture fissures
}

\author{
CHIHARU KOBA ${ }^{1}$, CHIHIRO KOGA $^{2}$, TAMAKI $\mathrm{CHO}^{3}$ and JINGO KUSUKAWA ${ }^{1}$ \\ ${ }^{1}$ Dental and Oral Medical Center, Kurume University School of Medicine, Kurume, Fukuoka 8300011; \\ ${ }^{2}$ Department of Oral and Maxillofacial Surgery, Center for Oral Diseases, Fukuoka Dental College; \\ ${ }^{3}$ Division of Biomedical Sciences, Department of Functional Bioscience, Section of Infection Biology, \\ Fukuoka Dental College, Kurume, Fukuoka 8140175, Japan
}

Received February 28, 2013; Accepted May 10, 2013

DOI: $10.3892 /$ br.2013.112

\begin{abstract}
With the growing number of elderly individuals, Candida is occasionally recognised as a fungal cause of aspiration pneumonia. In addition, there are numerous investigational reports on oral candidiasis. However, there are currently no reports on Candida contamination of denture base materials. This study was conducted to investigate Candida species in the oral cavity, denture parts and oral lesions of older/elderly subjects with oral candidiasis. The Candida strains were isolated and the species identified. Candida was also cultured in a medium with sample of denture resin and observed under an electron microscope. The results demonstrated the presence of several Candida species in the lesions of the oral mucosa and the surface and inner portions of the dentures. The following species of Candida were detected: Candida albicans, Candida glabrata, Candida tropicalis and Candida parapsilosis. Using electron microscopy, the invasion of Candida was observed in the incomplete polymerized resin base material and/or through microcracks (fissures) that have a tendency to form in used dentures. It was concluded that Candida may be present on the surface as well as the deeper portions of dentures. In addition, it appeared necessary to consider denture and oral cavity cleaning and the risks of remaking old dentures.
\end{abstract}

\section{Introduction}

Candida is a fungus that is indigenous to the oral cavity. It has been proven that it is the most frequently observed mycosis, carried by $15-60 \%$ of healthy individuals $(1,2)$. Although it may be present in the healthy organism it usually causes no disorders. However, following the long-term use of antibiotics, steroids or due to an overall decline in the immune system

Correspondence to: Dr Chiharu Koba, Dental and Oral Medical Center, Kurume University School of Medicine, 67 Asahi-machi, Kurume, Fukuoka 8300011, Japan

E-mail: koba_akihiro@kurume-u.ac.jp

Key words: Candida, denture resin, electron microscopy function, it may multiply opportunistically and become pathogenic (3).

Candidiasis may be classified as superficial or deep. Superficial candidiasis appears in the oral cavity, vaginal mucous membranes, skin and nails, whereas deep candidiasis appears in the gastrointestinal and urinary tracts, lungs, heart and cerebrospinal compartment. When severe, it may prove fatal. It is considered that superficially located Candida may reach every organ in the body via the blood stream or by way of the esophagus and trachea. In addition, the oral cavity may provide access to the alimentary tract and the lungs. Therefore, it is hypothesized that superficial Candida in the oral cavity may be the cause of deep candidiasis (4).

Previously, it was demonstrated that professional oral care prevents fever caused by pneumonia in the elderly (5). Furthermore, Kikuchi et al (6) demonstrated in an experiment on the elderly using isotopes that saliva from the oral cavity constantly flows into the lungs. Therefore, it is critical for the prevention of aspiration pneumonia to decrease the bacteria and fungi in the oral cavity through oral hygiene. Candida is one of the fungi that may cause aspiration pneumonia and there is a possibility that Candida in the oral cavity descends into the lungs and exacerbates pneumonia (7). As regards denture candidiasis, it is hypothesized that dentures and the multiplication of Candida are significantly associated. Consequently, it is considered significant from the viewpoint of oral care to elucidate the association between Candida and the fitting surface of the denture (8).

A therapeutic trial was performed using miconazole on a patient with dentures who suffered from oral candidiasis. A microbiological examination for Candida was conducted in the lesions, the inner side of the fitting surface of the denture and material from the deep portions of the denture, confirming the presence of Candida inside the denture material (9).

In the present study, to better understand candidal contamination of denture materials, an experiment was performed by adhering four species of Candida clinical strains onto samples of denture material. The strains had been presumptively identified in CHROMagar Candida culture medium (Chromaagar, Paris, France), which has good selective property and is able to easily identify fungal species $(7,10)$. It was proven using scanning electron microscopy that all four Candida species exhibited a tendency to adhere to the denture material. 


\section{Materials and methods}

Patients and specimens. The present study included 20 patients with dentures who underwent medical examinations at the Dental and Oral Medical Center, Kurume University Hospital. The patients provided written informed consent in order to participate in the study. The study protocol conformed to the ethical guidelines of the Declaration of Helsinki as reflected by prior approval by the Institutional Ethics Committee of the Kurume University School of Medicine. The patients presented with white spots on the mucosal membrane, which were diagnosed as oral candidiasis. The patients consented to sample collection from their dentures for our experiments.

The subjects included 9 males and 11 females. Their ages were as follows: 50-60 years, 1 patient; $60-70$ years, 2 patients; $70-80$ years, 5 patients; $80-90$ years, 8 patients; and $>90$ years, 4 patients. The average age of the subjects was 79.5 years. The main complaint of 11 subjects was a strange sensation in their mouth; 5 subjects complained of a burning sensation on their tongue; 2 complained of pseudomembrane formation; and the remaining 2 subjects complained of dental pain. In addition, all the subjects suffered from oral candidiasis. As regards other complications, 18 subjects had suffered cerebral infarction, 17 had diabetes, 14 had hypertension, 11 had heart disease, while other complications included fractures of the pelvis or the collarbone. The subjects in this study were mainly elderly individuals and had overlapping underlying diseases. There was no administration of oral antibiotics or use of steroids at the time of sample collection.

Diagnosis of oral candidiasis was confirmed following Candida detection in the lesions by microscopic examination. Sixteen of these cases involved the dorsum of the tongue, 2 involved the hard palate mucosa and the remaining 2 cases the buccal mucosa. There was no evidence of candidiasis on the upper or lower gums. As regards the type of dentures, our study was limited to hot polymerized resin-based dentures, complete or partial. Nine patients had both upper and lower dentures. One had only an upper denture and the remaining 10 had lower dentures.

\section{Experimental conditions}

Experiment 1: Collection of clinical Candida strains and identification of species

Sample collection. Samples were collected from three locations (lesions, inside the fitting surface and deep portions of the denture material) with the use of sterile swabs. As regards the lesions, 16 samples were collected from the dorsal mucosal membrane of the tongue, 2 from the hard palate mucosa and 2 from the buccal mucosa.

For lesions on the tongue or the buccal mucosa, 18 samples were collected from the nearest spot on the inside of the fitting surface of the lower denture. For lesions on the hard palate mucosa, 2 samples were collected from the fitting surface of the upper denture close to the axle of the tooth crest of the alveolar bone. In order to collect samples from the deep portions of the denture material the following procedure was performed: the denture was thoroughly washed with physiological saline solution. Subsequently, $\sim 1 \mathrm{~mm}^{3}$ of material was milled out using a round bur from the same location on the fitting surface where the previous samples had been collected (Fig. 1). The

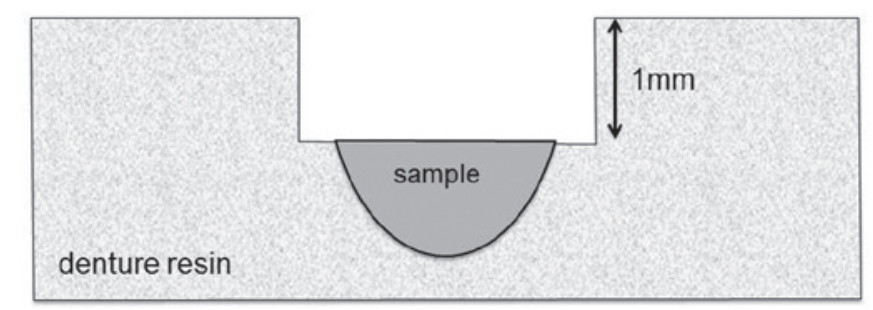

Figure 1. Collection of samples from the deep portions of the denture resin was performed as follows: the denture was thoroughly washed with physiological saline solution. Subsequently, at the same place on the fitting surface from which samples were collected, drilling was performed to a depth of $1 \mathrm{~mm}$ using a round bur, following which the denture was again washed. Then, $1 \mathrm{~mm}^{3}$ of material was removed from the drill site, the resin flakes were placed on sterile cotton swabs soaked with physiological saline solution and used as samples.

material was then placed on sterile cotton swabs soaked in physiological saline solution and used as a sample. The milled out material was replaced by new resin. It was decided that the collection of lesion and denture samples should be performed on 1 of our patients only.

Isolation of Candida. Isolation of Candida from the samples was performed with BBL ${ }^{\mathrm{TM}}$ CHROMagar ${ }^{\mathrm{TM}}$ Candida medium (Becton Dickinson, Sparks, MD, USA) $(10,11)$. This medium was selected since each colony develops a specific colorization that renders it readily identifiable. The samples were then inoculated into the medium and aerobically cultured for 3 days at $35^{\circ} \mathrm{C}$.

Presence or absence of Candida and identification of isolated strains. The presence of Candida was determined by counting the number of colonies and then classifying them into 3 levels as follows: a, (1-100); b, (101-1,000); and c, $(\geq 1,001)$. For the identification of isolated strains, the API 20C Auxanogram kit (Analytab Products, Inc., Systems SA, Marcy l'Etoile, France) was used and the fungal species were identified by their biochemical characteristics. The API 20C Auxanogram is a system consisting of 20 microtubes on a single plate with 19 dehydrated substrates used in biochemical tests to identify the pathogenic yeast $(12,13)$.

The medium was diluted by physiological saline $\left(0.85^{\circ} \mathrm{C}\right)$ with 2 drops of fungal liquid suspended in a concentration of McFarland no. 2. It was then transferred by pipetting into the microtubes to the horizontal level and incubated for $48-72 \mathrm{~h}$ at $30^{\circ} \mathrm{C}$. The development of the fungus in each microtube was observed and identifications were made using the positive rate table and APILAB software. As regards low selectivity identification that required microscopic morphology for confirmation, a slide culture was conducted using Tween- 80 combined with Corn Meal Agar. Identification was based on these observations (14-16).

Experiment 2: Candida culture in the medium with sample of denture resin

Cultures of isolate strains with denture material. Sabouraud bouillon medium $(10 \mathrm{ml})$ was used as a multiplication medium in the test tubes. Samples were collected from the resin base of the dentures from 1 patient by $3 \times 5-\mathrm{mm}$ sections. For used dentures, 2 types of resin base samples were prepared, 1 with and 1 without a fissure. Four Candida species 


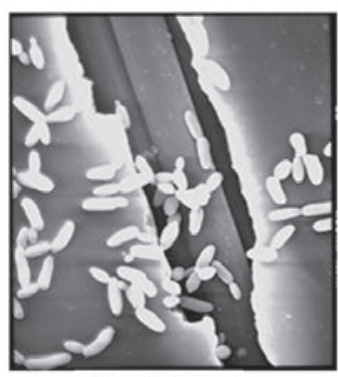

C.albicans

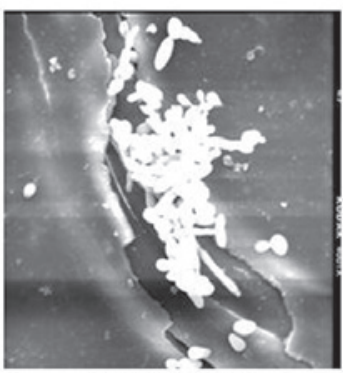

C.glabrata

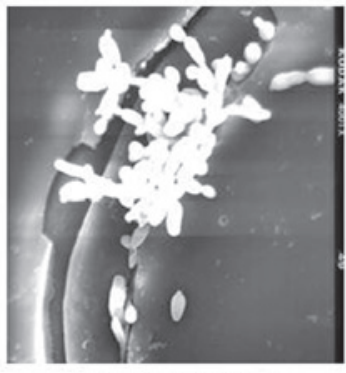

C. tropicalis

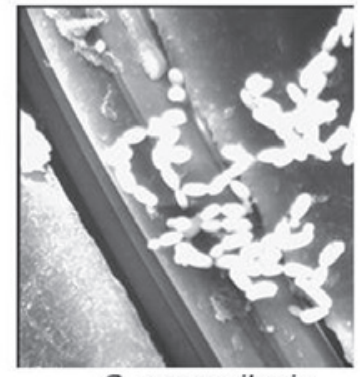

C.parapsilosis

Figure 2. Electron microscopic images of Candida albicans, Candida glabrata, Candida tropicalis and Candida parapsilosis adhering onto the surface of the denture. Original magnification, x1,000.

were used in this study, Candida albicans, Candida glabrata, Candida tropicalis and Candida parapsilosis. Two test tubes for each species were prepared by inoculating the Sabouraud bouillon medium with the fungal colonies. The two types of resin samples collected from the test denture material were placed into the test tube medium and incubated while shaking for $14 \mathrm{~h}$ at $35^{\circ} \mathrm{C}$. Prior to incubation the test tubes and resin samples were cleaned using an ultrasonic cleaner and sterilized.

Scanning electron microscope (SEM). To conduct the SEM observation, the resin samples were removed from the culture medium and fixed in $2 \%$ glutaraldehyde $(100 \mathrm{mM}$ phosphate buffer, $\mathrm{pH}$ 7.2) for $1 \mathrm{~h}$ at $4^{\circ} \mathrm{C}$. After washing in distilled water, the samples were fixed in $2 \%$ osmic acid solution $(100 \mathrm{mM}$ phosphate buffer, $\mathrm{pH} 7.2$ ) for $5 \mathrm{~min}$ at $4^{\circ} \mathrm{C}$. Subsequently, the resin samples were dehydrated through a graded series of ethanols $(50,60,70,80,90$ and $100 \%$ for 15 min each). The ethanol was replaced with tert-butyl-alcohol and the samples were freeze-dried for $12 \mathrm{~h}$. The samples were then capsule-coated with metal and used for scanning electron microscopic observation, which was performed with the S-800 SEM (Hitachi High-Technologies Corporation, Tokyo, Japan) (17-19).

\section{Results}

Positive rates of Candida. The number of colonies were classified as: a, (1-100); b, (101-1,000); and c, ( $\geq 1,001)$ and the association was determined between the location from which the samples were collected, the positive rate of fungus detection and the isolated fungus. Irrespective of the species of isolated fungus, the presence of Candida was confirmed in all the samples from the lesions and the inner side of the denture fitting surface. The presence of Candida was also observed in $60 \%$ of the samples collected from the deeper portions of the denture material. The association between the isolated fungi and the locations where they were encountered were as follows: Candida albicans was the most common, being detected in $54 \%$ of the lesions, $37 \%$ of the inner sides of the fitting surfaces and $39 \%$ of the deep portions of the denture base materials. The number of colonies was $<100$, which was quite limited. Candida albicans ranked high among the other species in each of the locations where Candida was detected. All four species were present in all the samples collected from the lesions, regardless of the location.
Moreover, as regards the inner side of the denture fitting surfaces, Candida albicans was present in 51\% and Candida glabrata in $34 \%$ of samples. In the samples collected from the deep portions of the denture materials, Candida albicans was the most common with a detection rate of $61 \%$, whereas few other fungal species were identified (Table I).

Results of SEM. The electron micrographs of Candida albicans, Candida glabrata, Candida tropicalis and Candida parapsilosis adhering onto the surface of the denture are shown in Fig. 2. Yeasts of all four species of Candida, whose shapes were either round (bulb) or oval (elliptical) multiplied on the surface of the denture. No differences in the size or shape of the fungi were detected in the present study. Candida albicans was most frequently observed as budding yeast, as shown in the cross section of the fissure (Fig. 3A). In addition, in incomplete polymerized resin base material, i.e., in places presenting with the formation of micro-bubbles, Candida glabrata was detected (Fig. 3B). No other yeasts were identified.

\section{Discussion}

The health of elderly individuals may be compromised, rendering them susceptible to infection, particularly by intraoral bacteria and general infection may lead to aspiration pneumonia and respiratory infections $(5,6)$. Ichikawa et al $(20)$ investigated the association between oral hygiene and infectious diseases, such as the association between methicillin-resistant Staphylococcus aureus (MRSA), Helicobacter pylori and denture plaque.

Moreover, studies on the number of pharyngeal bacteria have been conducted in elderly and young populations. The results of these studies demonstrated that the numbers of Candida and blue pus bacillus were significantly higher among the elderly (21). During the early stage of plaque formation, the rate of adherence increases along with anaerobic bacteria, mainly Streptococcus. Furthermore, Candida and Staphylococcus increase proportionately in denture plaques (22). Therefore, it is hypothesized that oral hygiene decreases the overall number of bacteria and in particular Candida, blue pus bacillus and Staphylococcus, which have a relatively strong pathogenicity and may be the cause of opportunistic infectious diseases. Plaque formed by healthy flora is considered important (23-25). Notably, Kotani et al (26) 
Table I. Detection sites and rates for Candida and number of colonies detected for each of the four species $(C$. albicans, C. glabrata, C.parapsilosis, C. tropicalis). ${ }^{\mathrm{a}}$

Site [n/total (detection rate)]

\begin{tabular}{|c|c|c|c|c|c|c|c|c|c|}
\hline \multirow[b]{2}{*}{ Species } & \multicolumn{3}{|c|}{$\begin{array}{c}\text { Lesions } \\
20 / 20(100 \%)\end{array}$} & \multicolumn{3}{|c|}{$\begin{array}{l}\text { Inner fitting surface } \\
20 / 20(100 \%)\end{array}$} & \multicolumn{3}{|c|}{$\begin{array}{l}\text { Deep portions of denture resin } \\
\qquad 16 / 20(80 \%)\end{array}$} \\
\hline & $\mathrm{a}$ & $\mathrm{b}$ & $\mathrm{c}$ & a & $\mathrm{b}$ & $\mathrm{c}$ & $\mathrm{a}$ & $\mathrm{b}$ & $\mathrm{c}$ \\
\hline C. albicans & 14 & 3 & 0 & 16 & 2 & 0 & 9 & 2 & 0 \\
\hline C. glabrata & 8 & 1 & 1 & 11 & 1 & 0 & 4 & 1 & 0 \\
\hline C. parapsilosis & 2 & 0 & 0 & 2 & 0 & 0 & 0 & 0 & 0 \\
\hline C.tropicalis & 1 & 1 & 0 & 2 & 1 & 0 & 1 & 1 & 0 \\
\hline
\end{tabular}

${ }^{a}$ The numbers of colonies are classified into the following categories: a, (1-100); b, (101-1000); and c, ( $\left.\geq 1001\right)$. Several species of Candida were detected in each sample.

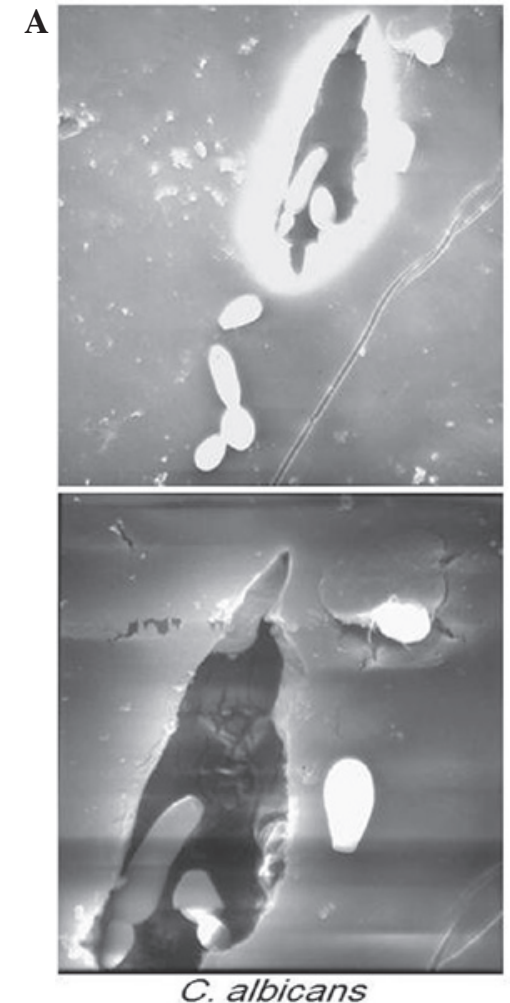

C. albicans

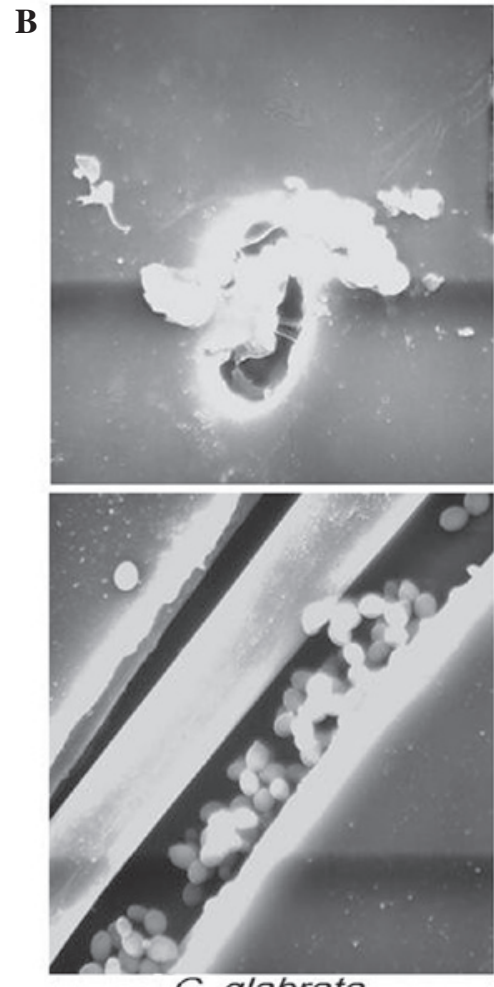

C. glabrata

Figure 3. (A) Candida albicans was most frequently observed as budding yeast, as shown in the picture of the cross section of the fissure. (B) Candida glabrata was observed in the microdefects in the polymerized resin base material. Original magnification, x1500.

reported that the number of Candida is an index that reflects the level of the condition of oral hygiene.

As demonstrated by our results, although Candida was detected in all the lesions, its detection rate was $55 \%$ in the samples collected from the inner side of the fitting surface of the upper denture and 60\% in those from the lower denture. As regards the samples collected from the deep portions of the resin material of the denture, Candida was detected in $45 \%$ of samples from the upper denture material and in $20 \%$ of samples from the lower denture material. According to previous studies, Candida albicans strongly adheres to resin, the base material of dentures and forms a biofilm which then becomes the foundation for denture plaque formation $(27,28)$. It was hypothesized that the Candida detected on the inner side of the denture was caused by denture plaque that adhered to the denture base material. However, in the case of Candida detected in the deep portions of the denture, this hypothesis seemed unlikely. Consequently, it was apparent that Candida existed deep within the resin base material. Therefore, we conducted Experiment 2 using an electron microscope. Since 
it is difficult to examine dentures that are actually being used, Candida was cultured onto the resin surface of used dentures and the cultures were used in our investigation.

The presence of all four species of Candida (yeast) on the resin surface was confirmed through the use of the electron microscope. However, Candida was not detected in the solid resin. In the Candida albicans medium, yeast was observed on incomplete polymerized resin base material, or where microbubbles were present. In addition, the presence of yeast was detected where the resin was cracked. These results suggest that, although Candida does not penetrate the resin, Candida invasion may result from the formation of microbubbles during the making of the denture or through seams created during repairs, enabling Candida to exist in the state of yeast. Dentures which have been in use for a long period of time may have undergone multiple repairs or have microbubbles in the resin base formed during the making process, potentially allowing Candida to penetrate and reside deep inside the resin. Therefore, it seems necessary to re-examine the cleaning methods used, in order to improve oral hygiene (29).

In addition, all the samples that were observed under an electron microscope during our study demonstrated a state of growth without threads. However, there was a previous study on electron microscopic images showing Candida with fungal threads in softened dentine and invading deeply into the dental canaliculi (30). Additional investigations are required, since there exists a possibility of obtaining different results from media resembling saliva and interstitial fluid $(31,32)$.

\section{Acknowledgements}

The authors gratefully thank Professor emeritus Tadamitu Kameyama for excellent experimental advice and support.

\section{References}

1. Barlow AJ and Chattaway FW: Observations on the carriage of Candida albicans in man. Br J Dermatol 81: 103-106, 1969.

2. Samaranayake LP: Oral candidosis: an old disease in new guises. Dent Update 17: 36-38, 1990

3. Epstein JB: Oral and pharyngeal candidiasis. Topical agents for management and prevention. Postgrad Med 85: 257-258, 263-265, 268-269, 1989

4. Arendorf TM and Walker DM: Oral candidal populations in health and disease. Br Dent J 147: 267-272, 1979.

5. Rose HD and Sheth NK: Pulmonary candidiasis. A clinical and pathological correlation. Arch Intern Med 138: 964-965, 1978.

6. Kikuchi R, Watabe N, Konno T, Mishina N, Sekizawa K and Sasaki H: High incidence of silent aspiration in elderly patients with community-acquired pneumonia. Am J Respir Crit Care Med 150: 251-253, 1994

7. Yoneyama T, Yoshida M, Matsui T and Sasaki H: Oral care and pneumonia. Oral Care Working Group. Lancet 354: 515, 1999.

8. Kurokawa H, Mizuguchi S, Takeda S, Nakamura T and Takanashi T: Relationship between oral mucosal lesions and the detection of Candida. Jpn J Oral Diagn/Oral Med 15: 26-30, 2002.

9. Nakamura C, Koga C, Kuhara S, et al: Clinical study of miconazole gel for Candida stomatosis of denture wearing patients. Shikai Tenbou 97: 1117-1123, 2001.

10. Odds FC and Bernaerts R: CHROMagar Candida, a new differential isolation medium for presumptive identification of clinically important Candida species. J Clin Microbiol 32: 1923-1929, 1994.
11. Beighton D, Ludford R, Clark DT, et al: Use of CHROMagar Candida medium for isolation of yeasts from dental samples. J Clin Microbiol 33: 3025-3027, 1995.

12. Heelan JS, Sotomayor E, Coon K and D'Arezzo JB: Comparison of the rapid yeast plus panel with the API20C yeast system for identification of clinically significant isolates of Candida species. J Clin Microbiol 36: 1443-1445, 1998.

13. Schuffenecker I, Freydiere A, de Montclos H and Gille Y: Evaluation of four commercial systems for identification of medically important yeasts. Eur J Clin Microbiol Infect Dis 12: 255-260, 1993.

14. Deak R, Bodai L, Aarts HJ and Maraz A: Development of a novel, simple and rapid molecular identification system for clinical Candida species. Med Mycol 42: 311-318, 2004.

15. Dealler SF: Candida albicans colony identification in 5 minutes in a general microbiology laboratory. J Clin Microbiol 29: 1081-1082, 1991.

16. Crivori P, Morelli A, Pezzetta D, Rocchetti M and Poggesi I: Development and validation of in silico models for estimating drug preformulation risk in PEG400/water and Tween80/water systems. Eur J Pharm Sci 32: 169-181, 2007.

17. Vitkov L, Krautgartner WD, Hannig M, Weitgasser R and Stoiber W: Candida attachment to oral epithelium. Oral Microbiol Immunol 17: 60-64, 2002.

18. Wilborn WH and Montes LF: Scanning electron microscopy of oral lesions in chronic mucocutaneous candidiasis. JAMA 244: 2294-2297, 1980.

19. Radford DR and Radford JR: A SEM study of denture plaque and oral mucosa of denture-related stomatitis. J Dent 21: 87-93, 1993.

20. Ichikawa T, Terada Y, Hirota K, Miyake Y, and Hoshino K: Study of oral health care and general health of elderly. Nihon Shika Igakkai Zasshi 19: 75-80, 2000 (In Japanese).

21. Preston AJ, Gosney MA, Noon S and Martin MV: Oral flora of elderly patients following acute medical admission. Gerontology 45: 49-52, 1999.

22. Shinada K, Teraoka K, Asaka T, et al: Distribution of Candida species and mutans streptococci related to oral conditions in elderly persons. Kokubyo Gakkai Zasshi 64: 512-517, 1997 (In Japanese).

23. Thein ZM, Samaranayake YH and Samaranayake LP: In vitro biofilm formation of Candida albicans and non-albicans Candida species under dynamic and anaerobic conditions. Arch Oral Biol 52: 761-767, 2007.

24. Branting C, Sund ML and Linder LE: The influence of Streptococcus mutans on adhesion of Candida albicans to acrylic surfaces in vitro. Arch Oral Biol 34: 347-353, 1989.

25. Pereira-Cenci T, Deng DM, Kraneveld EA, et al: The effect of Streptococcus mutans and Candida glabrata on Candida albicans biofilms formed on different surfaces. Arch Oral Biol 53: 755-764, 2008.

26. Kotani H, Sadamori S, Nikawa H and Hamada T: Clinical survey on denture stomatitis. 1. Relation between denture plaque and denture stomatitis. Nihon Hotetsu Shika Gakkai Zasshi 33: 208-214, 1989 (In Japanese).

27. Vasilas A, Molina L, Hoffman M and Haidaris CG: The influence of morphological variation on Candida albicans adhesion to denture acrylic in vitro. Arch Oral Biol 37: 613-622, 1992.

28. Thein ZM, Samaranayake YH and Samaranayake LP: Characteristics of dual species Candida biofilms on denture acrylic surfaces. Arch Oral Biol 52: 1200-1208, 2007.

29. Samaranayake YH, Cheung BP, Parahitiyawa N, et al: Synergistic activity of lysozyme and antifungal agents against Candida albicans biofilms on denture acrylic surfaces. Arch Oral Biol 54: 115-126, 2009.

30. Sen BH, Safavi KE and Spangberg LS: Colonization of Candida albicans on cleaned human dental hard tissues. Arch Oral Biol 42: 513-520, 1997.

31. Nikawa H, Samaranayake LP, Tenovuo J, Pang KM and Hamada T: The fungicidal effect of human lactoferrin on Candida albicans and Candida krusei. Arch Oral Biol 38: 1057-1063, 1993.

32. Nikawa $\mathrm{H}$ and Hamada $\mathrm{T}$ : Binding of salivary or serum proteins to Candida albicans in vitro. Arch Oral Biol 35: 571-573, 1990. 